\title{
A Short-Term Wind Power Forecasting Approach using ANFIS
}

\author{
${ }^{1}$ Joseph N. Mathenge, ${ }^{2}$ David K. Murage, ${ }^{3}$ John N. Nderu \\ ${ }^{1} \mathrm{MSc}$ Student, Department of Electrical and Electronic Engineering, Jomo Kenyatta University of Agriculture and Technology \\ (JKUAT), Nairobi, Kenya \\ ${ }^{2,3}$ Professor, Department of Electrical and Electronic Engineering, Jomo Kenyatta University of Agriculture and Technology
}

(JKUAT), Nairobi, Kenya

\begin{abstract}
In recent times, there has been a growing penetration of wind power in electrical grids globally. On one hand, this has led to the reduction in the cost of unit power while on the other hand brought about the challenge of ensuring grid reliability in cases where the penetration of wind power is high. This is brought about by the high intermittency and limited predictability of wind power hence making it a non-dispatchable resource, unlike conventional power sources. As a result of this, there has been a parallel growth in the research around wind speed and wind power forecasting techniques to ensure that the maximum benefits of wind power are realized at the cheapest cost possible. Neural networks have been at the core of this research owing to their ability to learn, versatility in handling time-series data, and their strength in establishing non-linear relationships between input and output datasets. In this paper, a hybrid Adaptive Neuro-Fuzzy Inference System (ANFIS) is enhanced using previous hour wind power and wind speed data to improve its accuracy in short-term wind forecasting.
\end{abstract}

Keywords: Wind Power Prediction, Short Term Wind Forecasting, Neural Networks.

\section{INTRODUCTION}

One of the greatest challenges in present-day electrical grids is ensuring that generation is sustainable and that the generated power is not only environmentally friendly but also affordable and socially accepted [1]. The goal of reducing dependency on fossil fuels which are limited resources in nature has seen a paradigm shift from fossil-powered generators to the incorporation of renewable in electrical power generation. On top of the risk of getting depleted, fossil fuels also emit greenhouse gases that harm the environment leading to global warming effects that disrupt climate and weather patterns.

The need to reduce our carbon footprint in the environment has seen many power grids shift from fossil fuel-based sources of energy to cleaner options of energy. This, along with the need to ensure sustainable continuity of supply to meet the growing global demand for electrical energy, has led to the inclusion of more renewables in the power systems energy mix. Wind and solar energy are among the renewables that have attracted the greatest interest in power systems since they occur freely in nature and can be harnessed anywhere around the globe. However, the high intermittency and limited predictability of wind pose a technological challenge when it comes to incorporating wind power into the grid.

Wind power is only available when the wind is blowing and the control of wind turbine output is very limited compared with other conventional generators such as hydro and steam generators. Due to the fluctuating wind power injected into the power system, the operational reliability of the grid cannot be guaranteed using the conventional methods of determining the spinning reserve capacity. Extra spinning reserves are required to accommodate wind power especially in cases it is incorporated in bulk [2]. With improved prediction algorithms, the reliability of the grid can be guaranteed and the cost of extra reserve capacity to cater for wind fluctuation reduced.

\subsection{Renewables in Power Systems}

Among some of the greatest challenges in electrical grids is the incorporation of intermittent renewables in the energy mix. Energy crisis, global warming, and depletion of fossil fuels have seen the world lean towards renewables when it comes to powering electrical grids globally. Renewables occur freely in nature and are considered clean hence have become among the most preferred sources of energy. Whilst geothermal energy is limited to geographic locations lying within volcanic regions, wind and solar energy are not limited by geographic location. As of 2020 , only 29 countries globally were using geothermal energy for electricity generation while wind and solar energy are practically found in all grids around the world [3].

There has been a growing interest in wind and solar energy technologies by scholars and a substantial growth in the 
capacities of power from the two renewables being incorporated into the energy mix by power system operators. Between the two renewables, wind poses the greatest challenge when it comes to grid integration due to its high intermittency and variability. Consequently, its reliability as an autonomous source of energy is greatly reduced and has to be consumed in a hybrid mix with other energy sources to ensure power system reliability is sustained.

Wind power is highly dependent on weather, seasonal changes, geographical topology, the direction of wind speed, and even time of the day hence making it erratic to determine [4]. Accurate forecasting methods and tools, therefore, become a necessity for the successful integration of wind power in electrical grids to ensure the most optimal decisions are made when running the power system. A good forecasting tool ensures that the maximum potential of wind energy is achieved and helps determine, in advance, how much wind energy shall be injected into the grid to allow the remaining generation sources to fill up the deficit and meet the demand.

With the interconnection of electrical grids, it is easy to manage the unpredictability of wind power. However, with the growing penetration of wind power in the grids, it becomes too expensive to sustain reserves to cater to the unpredictability of this renewable source of energy. In addition to this, isolated grids such as those on islands or minigrids also face a big challenge in ensuring that generation follows demand more so where the renewables contribute to a significant share of the demand. When there is low wind power generation, other conventional sources of energy are engaged to meet the demand. When there is a high wind power generation, the conventional sources are reduced to ensure that the advantages of this free intermittent resource are enjoyed. For proper coordination of intermittent sources such as wind power and other sources of generation in the grid, there is the need for accurate prediction tools to allow for accurate planning to ensure that demand is met and there is no curtailment of wind power.

Wind power is playing a major role in modern-day power systems. With the continued penetration of wind-powered generation in electrical grids worldwide, the area of wind power prediction has attracted the attention of researchers. The world's cumulative installed wind capacity has doubled every 3 years in the last decade [5]; an indicator of the growing acceptance of this renewable technology. Wind is stochastic and its behavior is a local phenomenon affected not only by weather parameters such as temperature, pressure, and humidity but also by the topology of the region in which it blows. This makes it a difficult task to predict the amount of wind power that is generated for a given horizon of interest.

\subsection{Wind Speed vs Wind Power Relationship}

The wind power equation is derived from the equation for kinetic energy (K.E) given by:

$$
K . E=\frac{1}{2} m v^{2}
$$

Where: $m$ is the mass of air

$v$ is the velocity of wind given in $\mathrm{m} / \mathrm{s}$.

The mass of flowing air particles used to generate the kinetic energy is a function of time since air is continuously flowing and is given as:

$$
\frac{d m}{d t}=\rho(t) A v
$$

Where: $\rho(t)$ is the density of air inkg $/ \mathrm{m}^{3}$. $A$ is the area swept by the wind turbine blades in $m^{2}$.

From equation (1), average electrical power $\left(P_{a v}\right)$ in Watts developed by wind is given as:

$$
P_{a v}=\frac{1}{2} \frac{d m}{d t} v^{2}
$$

Substituting equation (2) in (3) we have:

$$
P_{a v}=\frac{1}{2} \rho A v \cdot v^{2}=\frac{1}{2} \rho A v^{3}
$$

The relationship between $P_{a v}$ and $v$ is seen to be an exponential relationship where for every change in $v, P_{a v}$ changes by a magnitude of $v^{3}$.

The graph below illustrates the relationship between $P_{a v}$ and $v$, as well as wind turbine operational limits, is as shown in the figure below:

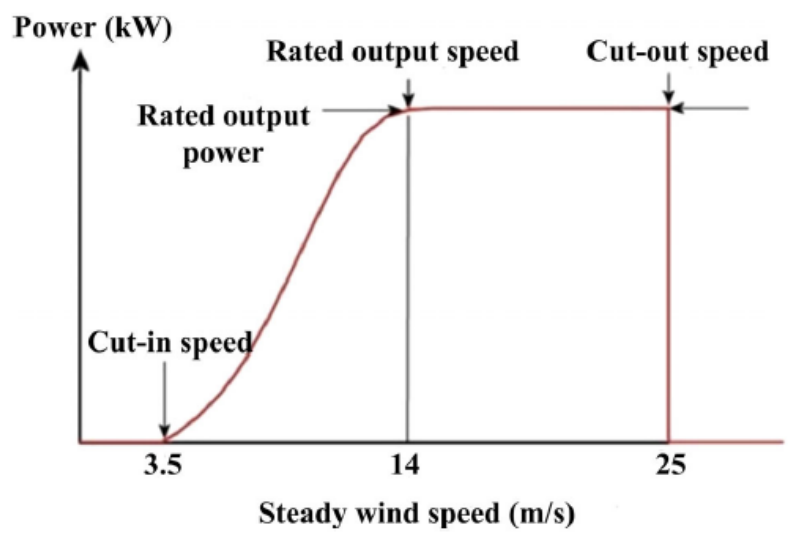

Figure 1: A Graph of Wind Power against Wind Speed

Not all available power can be extracted from the wind and hence to get the realistic power $P$ (Watts), $P_{a v}$ is multiplied by a coefficient as shown in the equation below: 
ISSN (online): 2581-3048

$$
P=C_{P} P_{a v}
$$

Where $C_{P}$ is the power coefficient or the coefficient of performance. It has an upper limit referred to as the Betz Limit equal to 0.593. Wind turbines cannot exceed an energy conversion efficiency of more than 59.3\% [5]. In practice, the Betz limit is found to be lower than 0.593 and helps cater for mechanical and electrical losses when converting wind speed to wind power. The power coefficient value is obtained from the manufacturer's data for a given wind turbine.

In [6], Asis Sarkar and D.Behera take the value of the power coefficient to be 0.593 but introduce two other multipliersto the wind power equation i.e. $N_{g}$ and $N_{b}$ which are the generator efficiency and gearbox efficiency respectively. These two constants cater for the electrical and mechanical losses that occur during the conversion of energy; an indicator to further confirm that not all energy from the wind can be converted into electrical power.

The density of air is affected by the relative humidity of the air, ambient temperature, and pressure of the air. Hence, these weather parameters also have an indirect impact on the amount of power that shall be produced by a given wind turbine. These variables can therefore be used in research to determine whether they help improve the wind power forecasts for a given horizon of interest.

\subsection{Wind Forecasting Timeframes}

There are four forecast horizons when considering the wind prediction problem as tabulated below [7]:

Table 1: Wind Speed/Power Forecast Timeframes

\begin{tabular}{|c|l|l|l|}
\hline \multicolumn{2}{|c|}{ Forecast Horizon } & $\begin{array}{c}\text { Prediction Ahead } \\
\text { Duration }\end{array}$ & \multicolumn{1}{c|}{ Applications } \\
\hline 1 & Very short term & $\begin{array}{l}\text { Few seconds to 30 } \\
\text { mins }\end{array}$ & $\begin{array}{l}\text { Real-time grid } \\
\text { operation decisions } \\
\text { and regulation, } \\
\text { market clearing, and } \\
\text { turbine control }\end{array}$ \\
\hline 2 & Short term & 30 mins to 6 hours & $\begin{array}{l}\text { Load dispatch } \\
\text { planning and load } \\
\text { intelligent decisions }\end{array}$ \\
\hline 3 & Medium Term & 6 hours to 1 day & $\begin{array}{l}\text { Operational security } \\
\text { in the electricity } \\
\text { market online and } \\
\text { off-line generating } \\
\text { decisions }\end{array}$ \\
\hline
\end{tabular}

The boundaries of these timeframes are however bound to different interpretations by different power system operators based on the applications they intend to utilize them for. Short-term planning could extend from 30 mins to 1 day and medium-term categorized as 1 day to several days ahead.

\subsection{Approaches to Wind Forecasting}

There are four approaches to wind power prediction:

1. Persistence method - This method assumes that the power in a future time shall be equal to the present measured power. This method is simple and economical and no external variables are needed to determine the output power. However, the accuracy of this method quickly deteriorated with an increase in the prediction horizon.

2. Physical approaches - This method used detailed physical characterizations to come up with the wind farm power model. It considers the numerical weather prediction data and the physical topology of the location of the wind farm. All these variables are then used in complex mathematical models which are highly time-consuming to determine wind speed. Using the manufacturer's wind power curve, the expected wind power can then be determined from the forecast wind speed. This method only relies on physical data and does not need to be trained with any historical data. This method is considered complex and one that requires a lot of computational resources [7] and is best suited for medium and long-term wind power prediction.

3. Statistical approach - These methods generally try to determine the linear and non-linear relationships between numerical weather prediction data and the corresponding generated power. To establish these relationships the model is trained using historical data. The model can further be tuned by comparing the model prediction and the online measured wind power. The model can then be used to predict wind power for the next few hours. Statistical methods can further be split into time series and neural networks subclasses.

(a) Time series models

These models were proposed by Box-Jenkins and apply historical data to come up with a mathematical model. The general form of the model is described as:

$$
X_{t}=\sum_{i=1}^{p} \varphi_{i} x_{t-1}+\alpha_{t}-\sum_{j=1}^{q} \theta_{j} \alpha_{t-j}(6)
$$

Where:

$\varphi_{i}$ represents the autoregressive parameter

$\theta_{j}$ is the moving average parameter

$\alpha_{t}$ is the white noise 
ISSN (online): 2581-3048

$p$ is the order of autoregressive

$q$ is the order moving average model

$x_{t}$ is the forecast wind power at time $\mathrm{t}$

Equation (6) represents the Auto Regressive Moving Average (ARMA) but if the value $p$ is assumed to be equal to zero, it shall represent the Moving Average (MA) model. Statistical methods based on this approach perform well in short-term wind power forecasting problems [7].

\section{(b) Artificial Neural Networks (ANNs)}

The ANN is the most commonly used method of wind power prediction due to its ability to identify non-linear relationships between input and output features presented to it. The black-box approach of the ANN helps avoid the complexity of the mechanics revolving around extraction and conversion of the kinetic energy from the wind into electric power.

The ANN maps random input vectors to their corresponding outputs by determining hidden patterns and relationships in historical data hence allowing the model to learn how to predict future output values.

An ANN consists of an input layer, hidden layer(s), and an output layer. It consists of processing units known as neurons which are connected through some weighted connections. It is the adjustment of the weights of these interconnections that we refer to as training of the neural networks. For the wind power prediction problem, since the desired output from the data is known, the type of training for this case is supervised training.

The performance of the ANN is dependent on the quality of the data used in training, the learning method used and the weight connections between the input and output data. There are more than 50 unique ANN variants in existence [7]. Some of those that have been used in wind power prediction include the Multilayer Perceptron (MLP), wavelet neural network (WNN), Back Propagation Neural Network (BPNN), radial basis function neural network (RBFNN), the convolutional neural network (CNN), the long short term memory (LTSM) among others.

4. Hybrid Approach - In the hybrid approach, combinations of different forecasting methods are used. The aim is to retain the best traits of one given method or approach and improve the accuracy of the suggested hybridized model.

\subsection{Related Work}

Short term wind power forecasting is critical when it comes to grid operation and management. An accurate shortterm forecast helps make decisions that ensure that grid reliability is guaranteed and helps make informed decisions in market-based ancillary services [5]. Wind is stochastic and it is also a localized phenomenon hence making predicting wind power a complicated task.

Basaran Filik and Tansu Filik in [8] analyzed three different models of predicting wind speed using ANNs. The first model used historical wind speed as the input; the second used historical wind speed and temperature as input while the third had historical wind speed, temperature, and pressure as inputs. Here, a two-layer feed-forward back propagation neural network was used. The training method chosen was the Levernberg-Marquardt due to its fast convergence times and the transfer function used was the log sigmoid. The data used had 20-second intervals and spans 30 days with the last 5 days used as testing data. Wind was forecast for 30 hours and 90 hours lead times with the results of the three models tabulated as below:

Table 2: RMSE and MAE performance for ANN Model in $[8](\Delta=30)$

\begin{tabular}{|c|l|l|l|}
\hline \multicolumn{4}{|c|}{ Lead time $=30$ hours } \\
\hline Model\# & Prediction Variable(s) & RMSE & MAE \\
\hline 1 & Wind speed & 0.6508 & 0.5046 \\
\hline 2 & Wind speed + temperature & 0.6502 & 0.5040 \\
\hline 3 & $\begin{array}{l}\text { Wind speed+ temperature+ } \\
\text { pressure }\end{array}$ & 0.6494 & 0.5032 \\
\hline
\end{tabular}

Table 3: RMSE and MAE performance for ANN Model in $[8](\Delta=90)$

\begin{tabular}{|l|l|l|l|}
\hline \multicolumn{4}{|l|}{ Lead time $=90$ hours } \\
\hline Model $\#$ & Prediction Variable(s) & RMSE & MAE \\
\hline 1 & Wind speed & 0.6940 & 0.5537 \\
\hline 2 & Wind speed + temperature & 0.6903 & 0.5470 \\
\hline 3 & $\begin{array}{l}\text { Wind speed+ temperature+ } \\
\text { pressure }\end{array}$ & 0.6759 & 0.5360 \\
\hline
\end{tabular}

It was observed that using more weather parameters to forecast the wind speed enhances the quality of the forecast by reducing the forecast error. The $3^{\text {rd }}$ model which had wind speed, temperature, and pressure as the inputs had the lowest RMSE and MAE in all cases. Also, as the lead time of the forecast increases, the forecast error increases showing that ANNs are best suited for shorter-term forecasts.

Aubai Alkhatib et al in [9] note that researchers rely on trial and error and some personal experience when it comes to designing and sizing a neural network for a given problem or dataset. Through trial and error, a feed-forward neural network with two hidden layers was settled upon and back propagation was used as the training algorithm. The versatility of the ANN in time series problem solving was again noted when compared with the older conventional methods. Anwen Zhu et al in [10] used the CNN in wind power prediction. With a dataset consisting of 35000 normalized data observations, the CNN gave an MSE of 0.00076 and 0.00078 for a forecast 
ISSN (online): 2581-3048

period of 4 hours ahead (16 data observations). The ability of neural networks to deal with large datasets was again observed.

In [11], Tarlochan Kaur et al highlight that the Central Electricity Regulatory Commission of India suggests that any wind power forecast should be atleast $70 \%$ accurate for it to be acceptable. Tarlochan et al use the ANN for wind speed prediction. Historical wind speeds at times $t, t+20$, and $t+40$ are used to predict wind speed for time $t+60$. Wind direction, temperature and humidity are also used as inputs to forecast the wind speed. After comparing 5 ANN models with various hidden layers and input neurons, a model with one hidden layer and 19 neurons in the hidden layer was realized to be the best fit and returned an MSE of 1.13 on the testing data.

G.J. Osorio et al in [12] use a hybrid model incorporating wavelet transform, evolutionary particle swarm optimization, and ANFIS to forecast wind speed for 3 hours ahead at 15minute intervals. The average MAPE was $3.75 \%$ with an average error variance of 0.0013 and outperformed ARIMA and Neural Network models. In [13] Ernesto Perez et al used ANFIS for short-term wind speed prediction. The generalized bell function was used in the model due to its flexibility and the prediction horizon was $16 \mathrm{hrs}$ to 48 hours. MSE values ranged from 1.568 to 1.633 and ANFIS was able to converge faster and had better accuracy than the ANN. Yordanos Kassa et al in [14] proposed an ANFIS model for short-term wind power prediction. The model was compared with Back Propagation Neural Network and Genetic Algorithm Neural Network and showed superior performance above the other two models with average MAPE values of $6.88 \%$. The review of previous works from various sources above serves to show the importance of hybridization in the wind forecasting problem to enhance performance. ANFIS being a hybrid model can outperform ANNs for all the evaluated works.

\section{PROPOSED MODEL}

In this paper, an ANFIS model to forecast wind power is proposed. The dataset contains hourly wind data observations spanning six years. Observations from one calendar year numbering 8760 points were used to predict the expected wind power for 24 hours ahead.

Evaluation of the dataset used revealed that wind speed and temperature had the best correlation with the wind power output. The correlation of wind direction and pressure against wind power was too low and did not add value to this prediction model. To further enhance the robustness of the suggested model, the previous hour wind speed and wind power historical values were used as inputs to determine the current wind power output with great results. The table below shows the correlation values between the selected input variables and our target variable (wind power).

Table 4: Correlation Values between Input and Target Variables

\begin{tabular}{|c|l|c|}
\hline \multicolumn{2}{|c|}{ Selected Input Variable } & $\begin{array}{c}\text { Correlation (r) with Target } \\
\text { Output Variable }\end{array}$ \\
\hline 1 & Wind Speed (current) & 0.9402 \\
\hline 2 & Wind speed (previous hour) & 0.8729 \\
\hline 3 & Temperature (current) & -0.2891 \\
\hline 4 & $\begin{array}{l}\text { Wind Power (previous } \\
\text { hour) }\end{array}$ & 0.9161 \\
\hline
\end{tabular}

These four variables were selected to be the input variables to predict the current wind power output. The high correlation between the previous hour wind power output and the present wind power output goes a long way to confirm the suitability of the idea behind the persistence model of short-term wind forecasting.

The following steps were followed in determining the wind power forecast:

a. Data acquisition and pre-processing.

b. Data standardization.

c. Statistical analysis of data to determine missing values and identify outliers.

d. Design of the ANFIS model and training.

e. Testing and tuning of the model.

The block diagram of the proposed model is as below:

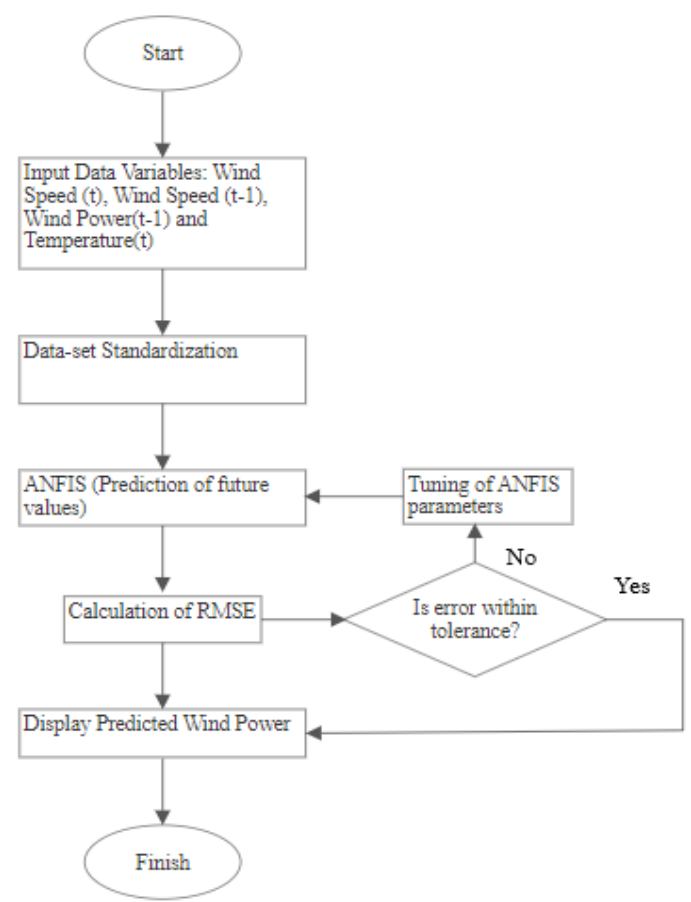

Figure 1: Block Diagram of Proposed Model 


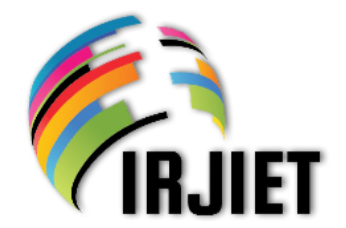

\section{RESULTS AND DISCUSSIONS}

The following results were obtained after training and testing the model to forecast wind power for a 24-hour timeframe. The test scenarios are listed below:

Scenario 1: ANFIS model trained with wind speed (t) Scenario 2: ANFIS model trained with wind speed $(\mathrm{t})$ and previous hour power $(\mathrm{t}-1)$

Scenario 3: ANFIS model trained with wind speed $(\mathrm{t})$, wind speed $(\mathrm{t}-1)$, temperature $(\mathrm{t})$, and wind power $(\mathrm{t}-1)$

3.1 ANFIS model trained with wind speed (t)

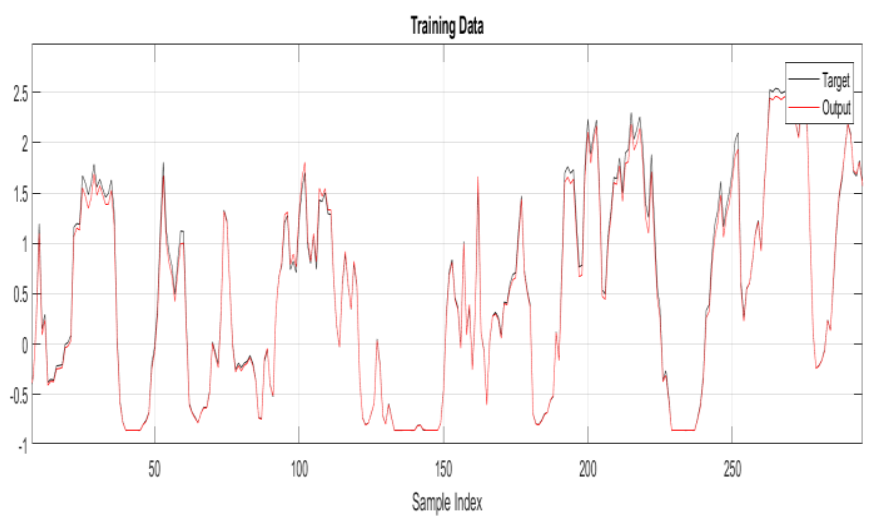

Figure 2: Graph of Training Data Target vs Model Output

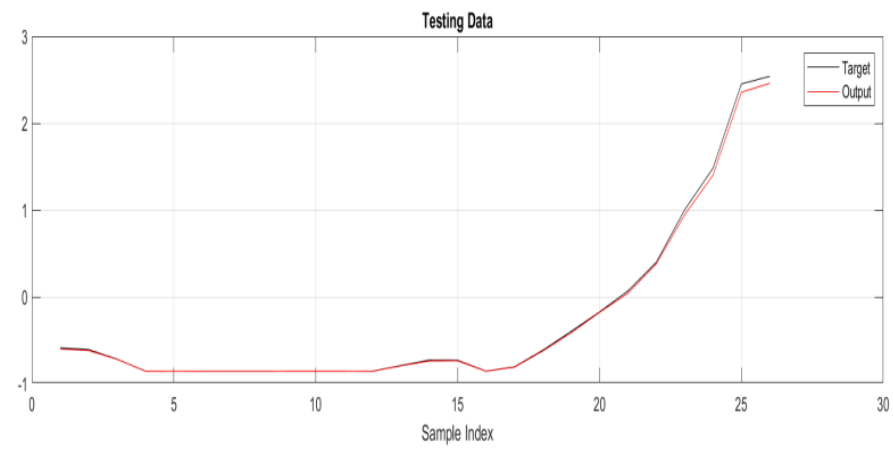

Figure 3: Graph of Testing Data Target vs Model Output

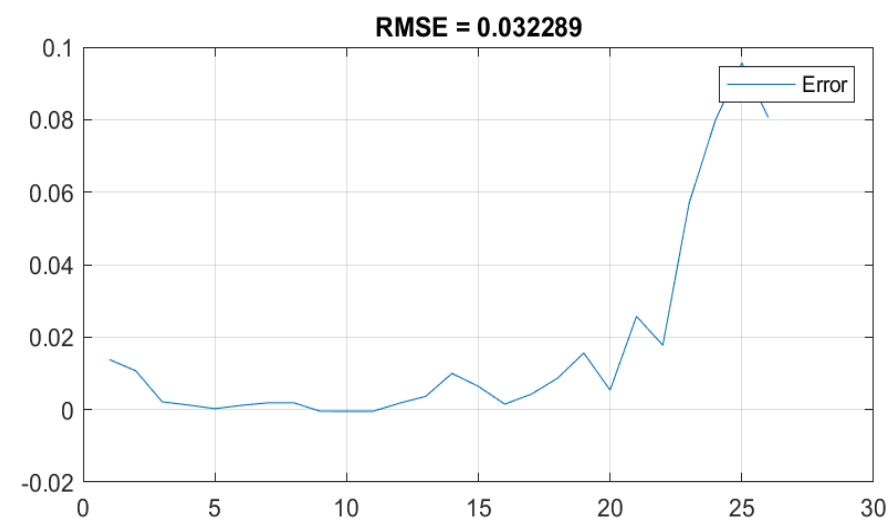

Figure 4: Graph of Average RMSE (Average RMSE=0.032289)
ISSN (online): 2581-3048

Volume 5, Issue 5, pp 35-42, May-2021 https://doi.org/10.47001/IRJIET/2021.505007

3.2 ANFIS model trained with wind speed (t) and previous hour power (t-1)

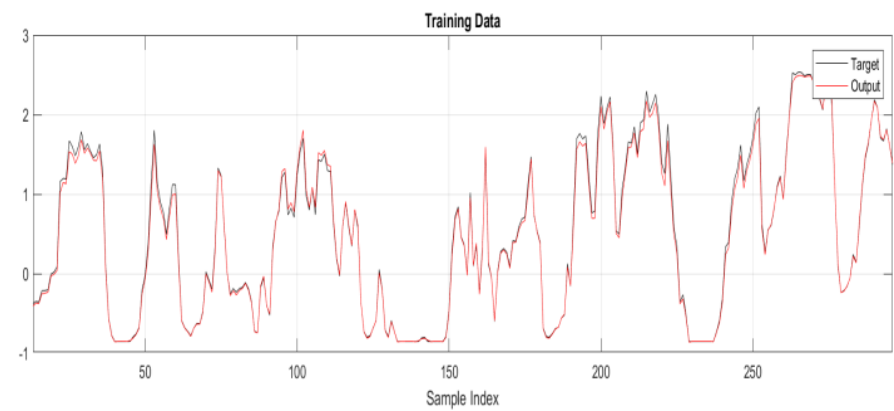

Figure 5: Graph of Training Data Target vs Model Output

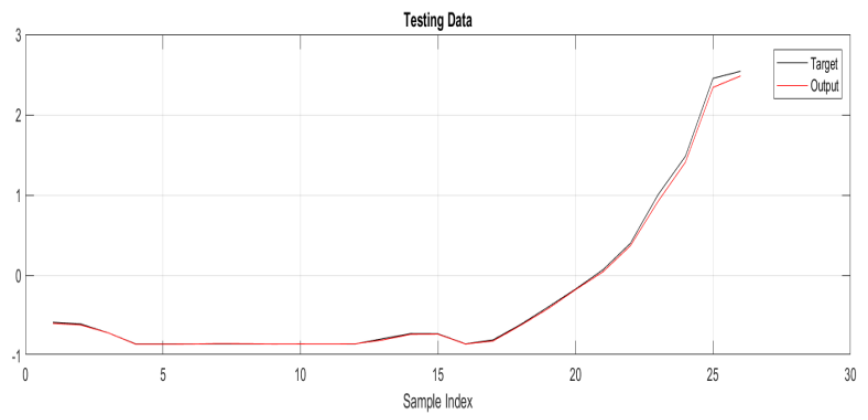

Figure 6: Graph of Testing Data Target vs Model Output

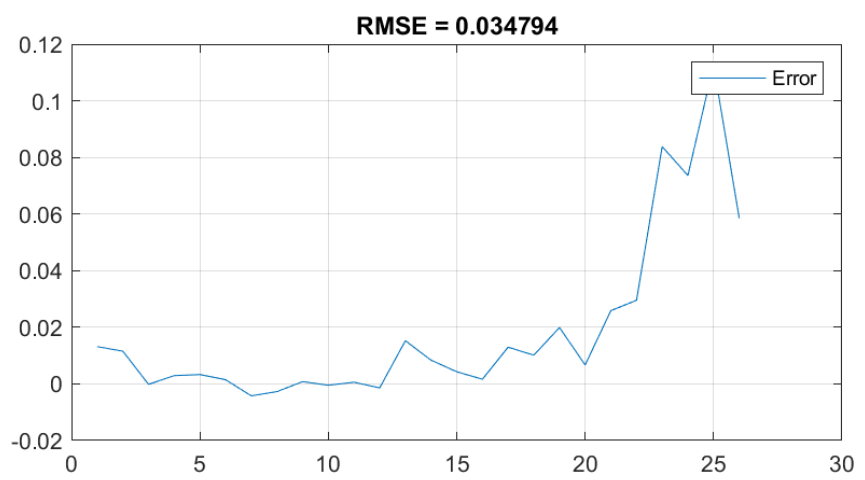

Figure 7: Graph of Testing Data RMSE (Average RMSE=0.034794)

3.3 ANFIS model trained with wind speed $(t)$, wind speed $(t-1)$, temperature $(t)$, and wind power $(t-1)$

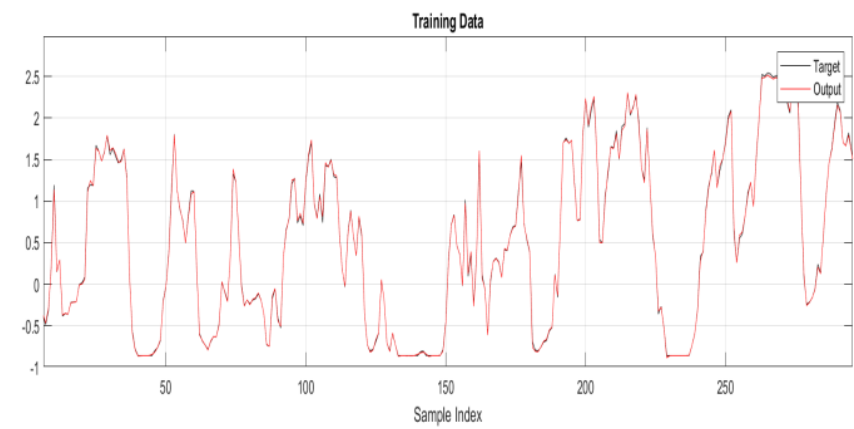

Figure 8: Graph of Training Data Target vs Model Output 
ISSN (online): 2581-3048

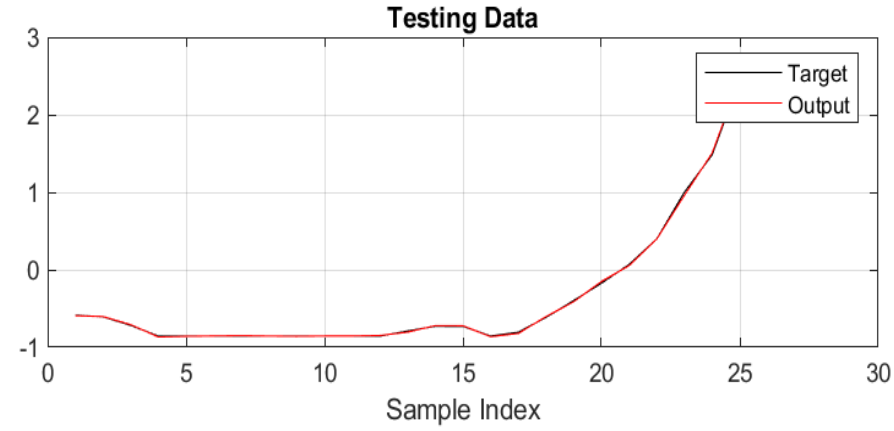

Figure 9: Graph of Testing Data Target vs Model Output

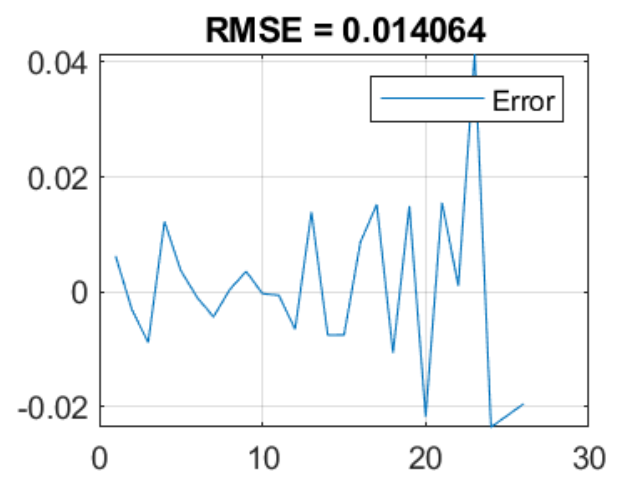

Figure 10: Graph of Testing Data RMSE (Average RMSE=0.014064)

3.4 Comparison of the RMSE values for the three test scenarios

Table 5: Comparison of Testing and Average RMSE Values for Three Test Scenarios

\begin{tabular}{|c|l|l|l|}
\hline \multicolumn{2}{|l|}{ Lead time $=24$ hours } & \\
\hline Model No. & Prediction Variable(s) used & $\begin{array}{l}\text { Testing } \\
\text { RMSE }\end{array}$ & $\begin{array}{l}\text { Average } \\
\text { RMSE }\end{array}$ \\
\hline 1 & Wind speed $(\mathrm{t})$ & 0.032289 & 0.044188 \\
\hline 2 & $\begin{array}{l}\text { Wind speed }(\mathrm{t})+\text { previous } \\
\text { hour power }(\mathrm{t}-1)\end{array}$ & 0.034794 & 0.038425 \\
\hline 3 & $\begin{array}{l}\text { Wind speed (t) +Wind } \\
\text { speed }(\mathrm{t}-1)+\text { temperature }(\mathrm{t}) \\
\text { previous hour power }(\mathrm{t}-1)+\end{array}$ & 0.014064 & 0.0155365 \\
\hline
\end{tabular}

From the results obtained above, it is clear that the inclusion of previous hour values of the previous hour variables helps enrich the wind power forecast. This proves that the logic behind the persistence model that the next hour forecast is assumed to be equal or close to the previous hour forecast holds and helps improve the forecast accuracy of ANFIS.

\section{CONCLUSION}

Time series prediction continues to be a very viable research area in power systems owing to the fact that most power system signals are non-linear in nature. The accuracy of wind power forecast models can further be improved by seeking to find patterns in historical datasets that help better future predictions. In this paper, previous hour data on power and wind speed proves very helpful in improving the accuracy of ANFIS in power prediction and should be extended and tested with other developed models and datasets.

\section{REFERENCES}

M. Altin, Ö. Göksu, R. Teodorescu, P. Rodriguez, B. B. Jensen, and L. Helle, "Overview of recent grid codes for wind power integration," Proc. Int. Conf. Optim. Electr. Electron. Equipment, OPTIM, pp. 1152-1160, 2010, doi: 10.1109/OPTIM.2010.5510521.

[2] Qiuwei Wu; Yuanzhang Sun, "Grid Code Requirements for Wind Power Integration," in Modeling and Modern Control of Wind Power, WileyIEEE Press, 2018, pp. 11-36.

[3] G. W. Huttrer, "Geothermal Power Generation in the World 2015-2020 Update Report," in Proceedings World Geothermal Congress 2020, 2020, p. 17.

[4] V. Prema and K. U. Rao, "Time series decomposition model for accurate wind speed forecast," Renewables Wind. Water, Sol., vol. 2, no. 1, p. 18, Dec. 2015, doi: 10.1186/s40807-015-0018-9.

[5] Q. Chen and K. A. Folly, "Comparison of Three Methods for Short-Term Wind Power Forecasting," in 2018 International Joint Conference on Neural Networks (IJCNN), Jul. 2018, pp. 1-8, doi: 10.1109/IJCNN.2018.8489472.

[6] D. K. B. Asis Sarkar, "Wind Turbine Blade Efficiency and Power Calculation with Electrical Analogy," Int. J. Sci. Res. Publ., vol. 2, no. 2, p. 5, 2012.

S. Hanifi, X. Liu, Z. Lin, and S. Lotfian, "A Critical Review of Wind Power Forecasting Methods-Past, Present and Future," Energies, vol. 13, no. 15, p. 3764, Jul. 2020, doi: 10.3390/en13153764.

[8] Ü. B. Filik and T. Filik, "Wind Speed Prediction Using Artificial Neural Networks Based on Multiple Local Measurements in Eskisehir," in Energy Procedia, Feb. 2017, vol. 107, pp. 264-269, doi: 10.1016/j.egypro.2016.12.147.

[9] A. A. S. H. M. Kurt, "Detailed Analysis for Implementing a Short Term Wind Speed Prediction Tool Using Artificial Neural Networks," Int. J. Adv. Networks Serv., vol. 5, no. 1, p. 10, 2012.

[10] A. Zhu, X. Li, Z. Mo, and R. Wu, "Wind power prediction based on a convolutional neural network," in 2017 International Conference on Circuits, Devices and Systems (ICCDS), Sep. 2017, pp. 131-135, doi: 10.1109/ICCDS.2017.8120465.

[11] T. Kaur, S. Kumar, and R. Segal, "Application of artificial neural network for short term wind speed forecasting," in 2016 Biennial International 
ISSN (online): 2581-3048

Volume 5, Issue 5, pp 35-42, May-2021

Conference on Power and Energy Systems: Towards Sustainable Energy (PESTSE), Jan. 2016, pp. 1-5, doi: 10.1109/PESTSE.2016.7516458.

[12] G. J. Osório, J. C. O. Matias, and J. P. S. Catalão, "Short-term wind power forecasting using adaptive neuro-fuzzy inference system combined with evolutionary particle swarm optimization, wavelet transform and mutual information," Renew. Energy, vol. 75, pp. 301-307, Mar. 2015, doi: 10.1016/j.renene.2014.09.058.

[13] V. H. G. R. Ernesto Cortés Pérez, Ignacio AlgredoBadillo, "Performance Analysis of ANFIS in short term Wind Speed Prediction," Int. J. Comput. Sci. Issues, vol. 9, no. 5, p. 9.

[14] Y. Kassa, J. H. Zhang, D. H. Zheng, and Dan Wei, "Short term wind power prediction using ANFIS," in
https://doi.org/10.47001/IRJIET/2021.505007

2016 IEEE International Conference on Power and Renewable Energy (ICPRE), Oct. 2016, pp. 388-393, doi: 10.1109/ICPRE.2016.7871238.

[15] J. Chen, W. Wu, B. Zhang, B. Wang, and Q. Guo, “A Spinning Reserve Allocation Method for Power Generation Dispatch Accommodating Large-Scale Wind Power Integration," Energies, vol. 6, no. 10, pp. 5357-5381, Oct. 2013, doi: 10.3390/en6105357.

[16] M. A. Ortega-Vazquez and D. S. Kirschen, "Estimating the Spinning Reserve Requirements in Systems With Significant Wind Power Generation Penetration," IEEE Trans. Power Syst., vol. 24, no. 1, pp. 114-124, Feb. 2009, doi: 10.1109/TPWRS.2008.2004745.

\section{Citation of this Article:}

Joseph N. Mathenge, David K. Murage, John N. Nderu, "A Short-Term Wind Power Forecasting Approach using ANFIS" Published in International Research Journal of Innovations in Engineering and Technology - IRJIET, Volume 5, Issue 5, pp 35-42, May 2021. Article DOI https://doi.org/10.47001/IRJIET/2021.505007 